\title{
Twenty-five-year-old Woman with Bilateral Borderline Ovarian Tumour Desir- ing to Preserve Fertility - Case Report and Literature Review on the Current State of Fertility Preservation in Women with Borderline Ovarian Tumours
}

\author{
25-jährige Frau mit beidseitigem Borderline-Tumor des Ovars und Wunsch nach Fertilitätserhalt - Fallbericht \\ und Literaturdiskussion über den aktuellen Stand der Fertilitätsprotektion beim ovariellen Borderline-Tumor
}

Authors

Affiliation
S. Findeklee, L. Lotz, K. Heusinger, I. Hoffmann, R. Dittrich, M. W. Beckmann

Department of Obstetrics and Gynaecology, Erlangen University Hospital, Erlangen, Germany

\section{Key words}

- borderline tumour

- fertility preservation

- future wish for child

- luteal phase stimulation

- ovarian cryopreservation

Schlüsselwörter

- Borderline-Tumor

- Fertilitätserhalt

- späterer Kinderwunsch

- Lutealphasenstimulation

- Kryokonservierung von

Ovargewebe und Eizellen

Deutsche Version unter: http://dx.doi.org/ $10.1055 / \mathrm{s}-0042-109267$

\section{received 9.1.2016 \\ revised $\quad 3.5 .2016$ \\ accepted 24.5.2016}

\section{Bibliography}

Dol http://dx.doi.org/

10.1055/s-0042-109267

Geburtsh Frauenheilk 2016; 76:

1189-1193 @ Georg Thieme

Verlag KG Stuttgart · New York

ISSN 0016-5751

Correspondence

\section{Dr Sebastian Findeklee}

Department of Obstetrics

and Gynaecology

Erlangen University Hospital

Universitätsstraße 21-23

91054 Erlangen

Germany

sebastian.findeklee@

uk-erlangen.de

\section{Abstract \\ $\nabla$}

Borderline ovarian tumours are semimalignant tumours occurring unilaterally or bilaterally with a peak incidence among women of reproductive age. Since the affected women often wish to preserve fertility, particular precautions must be taken when counselling the patient and obtaining consent prior to planning an individual treatment. Options for preserving fertility include an organ-sparing surgical procedure and cryopreservation of oocytes and/or ovarian tissue. In this article, we report on a 25 -year-old patient with a bilateral seromucinous borderline tumour who desired all fertility-preserving options. In order to perform the procedure without delay, we opted to perform luteal phase stimulation prior to oocyte retrieval. We conclude by discussing the current literature on the state of fertility preservation in the treatment of borderline ovarian tumours.

\section{Introduction}

$\nabla$

Around 10 to $20 \%$ of all epithelial ovarian tumours are serous or seromucinous borderline ovarian tumours. Their incidence is around 24 per 1000000 women [1]. The World Health Organization (WHO) defines borderline tumours as having both malignant characteristics (usually cellular atypicality) and benign characteristics such as the absence of invasive-destructive growth or stromal invasion. Borderline tumours exhibit a complex papillary architecture with at least 2 of the following characteristics:

- epithelial cell proliferation in the form of small papillae without a connection to the internal tissue)

- multilayered epithelium with atypicality (layers of atypical epithelial cells on the papillae)

\section{Zusammenfassung \\ $\nabla$}

Borderline-Tumoren des Ovars sind semimaligne ein- oder beidseitig auftretende Tumoren, die ihren Altersgipfel bei Frauen im reproduktiven Alter haben. Da die betroffenen Frauen häufig einen Kinderwunsch hegen, ergeben sich besondere Notwendigkeiten der Aufklärung vor der Planung einer individuellen Therapie. Möglichkeiten des Erhalts der Fertilität sind ein organschonendes operatives Vorgehen sowie die Kryokonservierung von Eizellen und/oder Eierstockgewebe. Wir berichten über eine 25-jährige Patientin mit beidseitigem seromuzinösem Borderline-Tumor, die alle Optionen des Fertilitätserhalts wünschte. Um die Operation zeitnah durchführen zu können, entschieden wir uns für eine Lutealphasenstimulation vor der Follikelpunktion. Abschließend diskutieren wir die aktuelle Literatur zum Stand der Fertilitätsprotektion beim ovariellen Borderline-Tumor.
- mitotic activity (increased mitoses or changes in the distribution of the mitoses)

- nuclear atypicality (increase in nucleus size and chromate density) [2]

A further peculiarity of this type of tumour is the fact that it is primarily diagnosed in women of reproductive age [3]. While $80 \%$ of borderline ovarian tumours involve only 1 ovary, around $20 \%$ occur bilaterally. The prognosis for bilateral involvement is less favourable [4]. While many authors propose an ablative surgical procedure with bilateral adnexectomy for treating early-stage borderline ovarian tumours, there are also indications that organ-sparing procedures that preserve fertility also have very good oncological outcomes. Fertility-preserving procedures include tumour enucleation, cystectomy and unilateral adnexectomy [5]. The disadvantages of this type of procedure are the higher risk of recurrence and need 


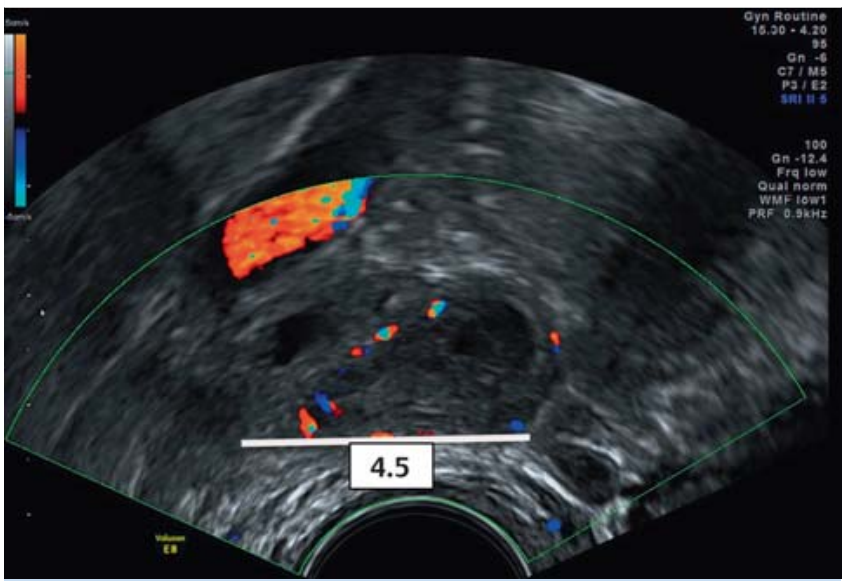

Fig. 1 Preoperative sonographic image of the right ovary.

for follow-up surgery. Other factors promoting a risk of recurrence are age $<30$, histology (micropapillary tumours have the least favourable prognosis) and tumour stage [6,7]. $\bigcirc$ Table 1 presents an overview of the tumour stages for borderline ovarian tumour, while Table 2 presents an overview of the histological subtypes and their characteristics. The decision for or against organ-sparing surgery depends on a variety of factors, including the patient's desire for fertility, age, gynaecological findings and the volume of ovarian tissue remaining [8]. If an organ-sparing surgical procedure is not an option despite the patient's current or future desire to become pregnant, for example, in the case of bilateral ovarian involvement or due to the disease progression, cryopreservation of oocytes after hormonal stimulation therapy and/or of ovarian tissue may be considered $[9,10]$.

\section{Case Report}

$\nabla$

We report on a 25-year-old woman (0 gravida) who first presented to our department on 12 October 2015 for consultation on fertility preservation after external diagnosis of bilateral seromucinous borderline ovarian tumour.

\section{Patient history}

Externally determined findings

The patient initially presented for urological examination in July 2015 due to lower abdominal pain of unknown origin. Based on the imaging results showing enlarged cystically changed ovaries bilaterally, gynaecological diagnostics were recommended. Subsequently, laparoscopic extirpation of an ovarian cyst was performed. Intraoperatively, extensive adhesions in the lower pelvis were observed, along with an approx. $3 \mathrm{~cm}$ pseudoperitoneal cyst in the area of the left ovary that ruptured spontaneously. Moreover, on the left ovary a lawn of roe-like deposits were observed that appeared brittle upon contact. The right ovary made a cystically changed impression, was $3 \times 4 \mathrm{~cm}$ in size and was otherwise unremarkable. In addition to adhesiolysis, the pseudoperitoneal cyst with a cystoid follicle on the left was removed, the tumour on the left was resected and the cyst removed. During histological examination, an atypically proliferated seromucinous borderline ovarian tumour was diagnosed bilaterally. The proliferation was low to moderate and focally pronounced, so that the risk of recurrence was deemed to be low. The 25-year-
Table 1 Tumour staging for borderline ovarian tumour.

\begin{tabular}{|c|c|}
\hline Tumour stage & Characteristics \\
\hline Stage I & Borderline tumour limited to the ovary \\
\hline Stage II & Tumour extension into the lesser pelvis \\
\hline Stage III & $\begin{array}{l}\text { Tumour extension below the lesser pelvis and/or } \\
\text { in the area of the retroperitoneum }\end{array}$ \\
\hline Stage IV & Distant metastases \\
\hline
\end{tabular}

Table 2 Histologic subtypes of borderline ovarian tumour [32].

\begin{tabular}{|c|c|}
\hline $\begin{array}{l}\text { Histologic } \\
\text { subtype }\end{array}$ & Characteristics \\
\hline Serous & $\begin{array}{l}\text { Papillary protuberances in the area of the internal } \\
\text { cyst wall } \\
\text { Low mitotic activity }\end{array}$ \\
\hline Mucinous & $\begin{array}{l}\text { Contains around } 50 \% \text { solid components } \\
\text { Mitotic activity only in exceptional cases }\end{array}$ \\
\hline Endometrioid & $\begin{array}{l}\text { Primarily solid } \\
50 \% \text { association with endometriosis } \\
\text { Low mitotic activity }\end{array}$ \\
\hline Brenner tumour & $\begin{array}{l}\text { Coarse stromal tumour up to several centimetres in size } \\
\text { with low mitotic activity }\end{array}$ \\
\hline $\begin{array}{l}\text { Clear cell- } \\
\text { micropapillary }\end{array}$ & Irregular tubuli with atypical clear cells \\
\hline
\end{tabular}

old woman was advised to undergo stage-appropriate surgery with a bilateral adnexectomy and resection of the greater omentum, the appendix and all macroscopically visible tumour foci. She was also advised to present to a second centre to obtain a second opinion on the histological findings. Since the young woman was planning to become pregnant in the future, she presented to our department for advice about fertility-preserving treatment options.

\section{Clinical findings}

At initial presentation, the patient reported that her overall state of health was good. The patient reported a regular menstrual cycle (28/3 days) and dysmenorrhoea. Apart from light smoking ( 2 cigarettes/day), there were no other remarkable aspects in the patient history. During the gynaecological examination, the only palpable abnormalities were a coarse resistance in the left adnexal area and in the ultrasonography, a prominent right ovary and little free fluid in the recto-uterine pouch ( $\bullet$ Fig. 1 ). We explained to the patient that the non-organ-conserving surgery proposed at the external facility was considered the standard treatment in the specific situation with respect to achieving maximum oncological certainty. At the same time, in view of the patient's explicit desire to preserve fertility, we discussed possible fertility-preserving options, including oocyte and ovarian tissue cryopreservation, as well as the attempt to spare an ovary and the uterus. We informed the patient about an increased risk of recurrence with this procedure, which, however, would not guarantee a future pregnancy. Our patient requested oocyte and ovarian tissue cryopreservation. We discussed hormonal stimulation therapy with oocyte cryopreservation as well as the subsequent surgical laparoscopy with bilateral tumour removal and unilateral ovarian tissue cryopreservation. The patient consented to a two-stage procedure comprising primary oocyte retrieval and 
preservation of biopsies and secondary stage-appropriate surgery in the event that the need for bilateral adnexectomy and, in the event of uterine involvement, hysterectomy, was determined intraoperatively. The patient presented to our endocrinological reproductive medicine out-patient department on 19 October 2015 to plan oocyte cryopreservation. Since the patient was on day 11 of her menstrual cycle, in consensus with the patient, we agreed to initiate experimental luteal phase stimulation starting on day 18 of the cycle (26 October 2015) to prevent a 14-day wait and thus counteract further dissemination of the tumour.

\section{Treatment}

We initiated hormonal stimulation therapy in the luteal phase on 26 October 2015 in the short antagonist protocol and our patient self-administered 200 (day 18 to 20 of the cycle)/150 IU FSH as a stimulation drug and the GnRH antagonist cetrorelix once a day. The stimulation lasted 16 days. During this period, we performed 3 sonographic check-ups with laboratory work-ups. The treatment resulted in the development of 3 follicles $>10 \mathrm{~mm}$ on the right and 1 follicle on the left. Two days prior to oocyte retrieval, the endometrium was well-established with $12.7 \mathrm{~mm}$. The estradiol value was $2126 \mathrm{pg} / \mathrm{nl}$. Our patient inducted ovulation with $0.2 \mathrm{mg}$ triptorelin. Oocyte retrieval was performed on 11 November 2015 and was uncomplicated. Cryopreservation of 5 unfertilised oocytes was subsequently undertaken. The 25-year-old woman then presented to our department for the planned surgical laparoscopy on 27 November 2015. Intraoperatively, the organs of the upper abdomen presented as unremarkable. In the lesser pelvis there were veil-like peritoneal adhesions in the left adnexa area. Both ovaries presented with cysts. We performed bilateral tumour extirpation and removed approximal one third of the right ovary for cryopreservation ( $\bullet$ Fig. 2). Since the other adnexa was unremarkable, we removed only the macroscopically visible tumour on the ovary. We also took several peritoneal biopsies in the area of the abdomen as a whole and performed an omentectomy. As there were no signs of peritoneal tumour dissemination, no other interventions were performed. The tumour dissemination corresponded with FIGO stage IC2. There were no perioperative or postoperative complications. The histological testing of the biopsies by our pathology department yielded a seromucinous borderline tumour in the area of the left ovary not related to the ovarian surface that had already been diagnosed by the referring facility. The right ovary showed tumour-free tissue with focal low-grade chronic inflammation. The peritoneal biopsy was also unremarkable. We discussed the results with our patient in detail and advised her to present for follow-up with rectovaginal palpation and ultrasonography every 3 months in the first 2 years post-surgery. The patient was advised to become pregnant soon after this period, no later than age 35 , in order to reduce the risk of recurrence. After this period, the patient was advised to present for follow-up on a 6-monthly basis up to 5 years post-surgery and on a yearly basis afterward. We also advised her to undergo bilateral adnexectomy and subsequent hormone replacement therapy after completion of childbearing.

\section{Discussion}

\section{$\nabla$}

The case report presented above describes a deviation from the standard treatment for bilateral ovarian seromucinous borderline tumour due to the individual situation of our 25-year-old patient's pronounced desire to become pregnant. In consensus with

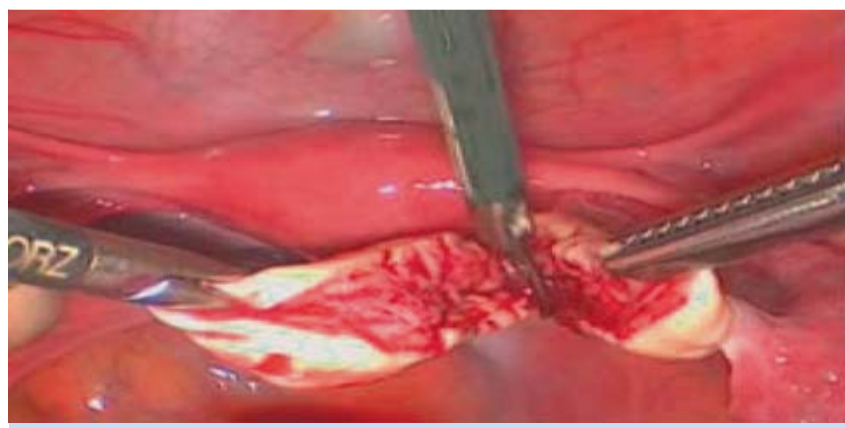

Fig. 2 Surgical site: Removal of a part of the ovary for cryopreservation.

our patient, we elected not to perform an adnexectomy and instead to employ an experimental treatment method with stageappropriate surgery and luteal phase stimulation. In the specific situation, the decision to perform the fertility-preserving surgery was particularly difficult in light of the fact that bilateral ovarian involvement and the performance of a cystectomy rather than an adnexectomy are definitively associated with increased risk of recurrence [19]. This also applies to a cystic rupture, which had occurred during the previous surgery performed at the referring facility. While the risk of borderline tumour recurrence is generally reported to be approx. 5\% after stage-appropriate surgery, this percentage can increase four-fold after fertility-preserving cystectomy such as that performed on our patient [11]. However, the literature also contains reports on the possibility of bilateral cystectomy in the case of bilateral ovarian involvement and explicit fertility-preserving desire. Vasconcelos et al. concluded that in this situation, there are no indications of increased recurrence rates compared to unilateral adnexectomy with contralateral cystectomy [12]. It should also be noted that the surgical procedure in this case complied with guidelines. The S3 guidelines for ovarian cancer state that if the patient wishes to have children and the tumour is limited to the ovary, a fertility-preserving procedure can be carried out [13]. This brings to light the fact that among young patients, oncological certainty and the wish to preserve fertility may be at odds with each other. For each situation, the attending physician needs to weigh the increased risk of recurrence against the gain in quality of life for the patient yielded by the ability to become pregnant in the future. This may cause an ethical dilemma for the physician that may be best resolved by addressing the topic of fertility directly with the patient. It is known that up to 1 in 7 patients would be prepared to take compromises in oncological certainty into account if it meant being able to have children later on [14]. At the same time, it is also known that as the patient's awareness of the significance of and options for preserving fertility increases, so does the frequency of educating the patients about this and in turn, the use of fertilitypreserving measures [15].

Nevertheless, the procedure continues to be a case-by-case decision that needs to be carefully considered by the young cancer patient. Zanetta et al. reported a recurrence rate of $18.5 \%$ for early disseminated cancer after fertility-sparing surgery as compared to a rate of only $4.7 \%$ after radical surgery. However, at the 70 month follow-up, the higher recurrence rate was not associated with a higher mortality. The rate of progression into invasive ovarian cancer has been reported at $2 \%[12,16]$. However, it must be critically noted that in up to $30 \%$ of all cases, the recurrence of a borderline tumour represents a classic invasive epithelial ovar- 
ian cancer with a much worse prognosis [17]. Thus, prior to taking the decision for or against a fertility-preserving procedure, it is indispensable to perform sufficient surgical staging including assessment of the internal genitalia, peritoneum and greater omentum, as was performed in our patient at the referring facility. It also includes the removal of multiple peritoneal biopsies in both the pelvis and the upper abdomen, as well as an omentectomy [17,18]. In 2013, Du Bois et al. published results similar to those reported by Zanetta et al. While the organ-sparing surgery was associated with a three-fold increase in the recurrence rate, the overall survival of the patients was not decreased [19]. The fact that the risk of mortality was not increased for patients experiencing recurrence of borderline tumours encouraged us to use the organ-sparing procedure. Thus, sparing the adnexa is a legitimate approach in the primary situation and a cystectomy is only absolutely necessary in the case of recurrence. We also informed our 25-year-old patient with the stage I borderline tumour about the recurrence rate of approx. 1:5 with the use of an organ-sparing procedure. The safety of hormonal stimulation therapy in patients with known borderline ovarian tumours is hotly debated. For example, in the late 1990s, Parazzini et al. reported a higher incidence of borderline tumours in women who had undergone hormonal stimulation therapy as part of infertility treatment [8]. All in all, very few studies focus on stimulation therapy in women with borderline tumours. In a study conducted by Denschlag et al. with 62 women and 152 treatment cycles, the recurrence rate of $19.4 \%$ after 52 months was comparable to the rate reported by Zanetta et al. [20]. Some other authors recommend that women with borderline tumours who are basically capable of spontaneous conception should wait 1 or 2 years before becoming pregnant, since most recurrences of borderline ovarian tumours occur within 24 months of treatment [7]. According to a study performed by Beiner et al., only 1 out of 7 women who underwent infertility treatment experienced a recurrence of a borderline tumour [21]. In a retrospective multicentric study from 2007, the risk of recurrence was reported as $1: 9$, with recurrence occurring only in women undergoing IVF treatment [22]. In vitro, no stimulatory effect of FSH or estradiol on the growth of borderline tumour cell cultures could be detected [23]. This may indicate that hormonal stimulation may be recommended in good faith for patients with borderline tumour who wish to preserve fertility. However, due to the sparse data available, the treatment should be used prudently after weighing the risks and benefits and only after the patient has been adequately informed.

Since our patient explicitly requested hormonal ovarian stimulation but was not at the beginning of her menstrual cycle when she presented to our endocrinological reproductive medicine out-patient department, after an in-depth consultation, we decided to initiate hormonal ovarian stimulation in the luteal phase starting on day 18 of the cycle in accordance with the stimulation protocol published by Wolff et al. [24]. In so doing, we weighed a potential compromise in oocyte quantity and quality against the risk of delaying the procedure, i.e. tumour cell dissemination. The low number of oocytes ultimately harvested, at only 5 , is however most likely due to the patient's low ovarian reserve after partial ovarian resection and tumour involvement of the ovarian tissue rather than to the stimulation protocol. One positive outcome is the fact that intraoperatively no involvement of the peritoneum was detected. Whether the more timely surgery was responsible for this cannot be conclusively assessed in retrospect. The literature contains several publications that describe luteal phase stimulation for preserving fertility in patients with cancer as an option with good results [25].

If our 25-year-old patient is unable to become pregnant, either spontaneously or by means of fertilisation of the cryopreserved oocytes with subsequent transfer, she will still have the option of transplantation of the resected ovarian tissue. The tissue can be implanted into the peritoneal pouch by means of laparoscopy and in $70 \%$ of cases it regains its function [9]. Since hormone production usually only occurs for a limited period, the patient should try to become pregnant very soon after the transplantation. In most cases, short-term ovarian stimulation, oocyte retrieval, in vitro fertilisation and subsequent embryo transfer are required. Worldwide, over 75 babies have been born thanks to this procedure. At present, the likelihood of women becoming pregnant who have undergone this procedure is around 25\% [26]. The method is therefore now the standard procedure for preserving fertility in women with cancer. Reasons for the seemingly low pregnancy rate include the challenge of creating a favourable ovarian milieu during transplantation, low ovarian reserve, often already prior to the procedure, and the as yet still limited experience with the relatively new procedure. If our patient eventually decides to undergo ovarian tissue transplantation, in addition to informing her about the general risks associated with the laparoscopic procedure, we will inform her about a potential risk of transplanting tumour cells. In the literature, this risk is reported to be around $1 \%$ across all tumour types and is highest for haematological neoplasms [27]. Due to the sparse number of cases similar to the case we have described here, there are no reports on statistics on the risk of transplanting malignant cells in patients with borderline ovarian tumour in the literature. However, for all neoplasms primarily involving the ovary an elevated risk must be assumed, that approximately coincides with the risk of recurrence in the case of the fertility-preserving procedure. In the case of recurrence, stage-appropriate surgery must be performed. Nevertheless, Fain-Kahn et al. reported that cryopreservation of ovarian tissue is possible in over half of patients with borderline ovarian tumour [28]. Other research groups also recommend removal and later transplantation of ovarian tissue for preserving fertility in women with a borderline tumour [9]. While the removal and later transplantation of ovarian tissue in general is no longer considered to be experimental, and is now the standard procedure for preserving fertility, in the specific situation of our patient, it must be considered to be an individual deviation from the generally recommended approach due to the elevated risk of ovarian tumour implants owing to the bilateral ovarian involvement [29]. In 2011, Lotz et al. analysed ovarian biopsies from 23 premenopausal women with epithelial and nonepithelial ovarian malignomas that were transplanted into SCID mice. Twenty-four weeks after transplantation of these biopsies, no malignant cells could be detected in the mice either histologically or under a light microscope [30]. Histological examination of the tissue can help lower the risk of transplanting tumour cells. Whether or not the patient wishes to become pregnant, regular check-ups are necessary during follow-up. Contrary to most other tumour types, a borderline ovarian tumour can still recur even 15 years after initial diagnosis [19]. We advised our patient to present for follow-up on a 3-monthly basis in the first 3 years post-surgery and then on a 6-monthly basis in the 2 years after that. Afterward, the patient should undergo life-long annual check-ups. While current debate focuses on the determination of tumour markers such as CA 125 and CA 19-9 in particular during follow-up, we do not perform these tests [31]. Since no adju- 
vant chemotherapy is required after borderline tumour surgery, we have advised the patient to become pregnant soon after surgery. After completion of childbearing, stage-appropriate surgery is planned.

\section{Conclusions}

\section{$\nabla$}

The attending physician's desire for maximum oncological certainty and the patient's need to preserve fertility in order to plan a pregnancy may be at odds during the treatment of malignant or semimalignant tumours. A special feature of semimalignant borderline ovarian tumours is that they often occur in young women who have not yet completed childbearing. For this reason, before conducting any cancer treatment, and especially in this particular situation, the patient should receive in-depth information from a physician with experience in oncofertility. If a patient is currently planning to become pregnant, she should be informed about an elevated postoperative risk of infertility due to the low ovarian reserve and possible tubal functional disorders, as well as about the elevated risk of recurrence, especially in the first 24 months post-surgery. If the ovarian reserve is normal and the spermiogram unremarkable, the patient can attempt to become pregnant 3 months post-surgery if she absolutely does not want to delay childbearing. If the woman wishes to become pregnant later, is without a partner or has diagnosed infertility, oocyte cryopreservation after prior hormonal stimulation therapy and/or ovarian tissue cryopreservation are conceivable options.

Our case report demonstrates the practical relevance of the issues associated with planning a pregnancy in women with cancer and presents possible options for action in clinical routine.

\section{Conflicts of Interest}

There are no conflicts of interest.

\section{References}

1 Harlow BL, Weiss NS, Lofton S. Epidemiology of borderline ovarian tumours. J Natl Cancer Inst 1987; 78: 71-74

2 Scully RE. World Health Organization. International Classification of Tumours. Histological Typing of ovarian Tumors. 2nd ed. Berlin, Heidelberg, New York, Tokio: Springer; 1999

3 Modugno F, Ness RB, Wheeler JE. Reproductive risk factors for epithelial ovarian cancer according to histologic type and invasiveness. Ann Epidemiol 2001; 11: 568-574

4 Nikrui N. Survey of clinical behavior of patients with borderline tumors of the ovary. Gynecol Oncol 1981; 12: 107-119

5 Patrono MG, Minig L, Diaz-Padilla I et al. Borderline tumours of the ovary, current controversies regarding their diagnosis and treatment. Ecancermedicalscience 2013; 7: 379

6 Uzan C, Kane A, Rey A et al. Outcomes after conservative treatment of advanced-stage serous borderline tumors of the ovary. Ann Oncol 2010; 21: 55-60

7 Daraï E, Fauvet R, Uzan C et al. Fertility and borderline ovarian tumour: a systematic review of conservative management, risk of recurrence and alternative options. Hum Reprod Update 2013; 19: 151-166

8 Parazzini F, Negri E, La Vet al. Treatment for fertility and risk of ovarian tumours of borderline malignancy. Gynecol Oncol 1998; 68: 226-228

9 von Wolff M, Montag M, Dittrich R et al. Fertility preservation in women-a practical guide to preservation techniques and therapeutic strategies in breast cancer, Hodgkin's lymphoma and borderline ovarian tu- mours by the fertility preservation network FertiPROTEKT. Arch Gynecol Obstet 2011; 284: 427-435

10 Findeklee $S$, Lotz L, Heusinger $K$ et al. Fertility protection in female oncology patients: how should patients be counseled? Geburtsh Frauenheilk 2015; 75: 1243-1249

11 Trillsch F, Mahner S, Ruetzel J et al. Clinical management of borderline ovarian tumors. Expert Rev Anticancer Ther 2010; 10: 1115-1124

12 Vasconcelos I, de Sousa Mendes M. Conservative surgery in ovarian borderline tumours: a meta-analysis with emphasis on recurrence risk. Eur J Cancer 2015; 51: 620-631

13 du Bois A, Petru E, Meier W et al. S3-Leitlinie Diagnostik, Therapie und Nachsorge maligner Ovarialtumoren, Juni 2013. Online: www.awmf. org; last access: 02.01.2016

14 Lee MC, Gray J, Han HS et al. Fertility and reproductive considerations in premenopausal patients with breast cancer. Cancer Control 2010; 17: $162-172$

15 Li A, Pasternak M, Duke C et al. The effect of practitioner education on fertility preservation awareness, perception and clinical practice. Obstet Gynecol 2015; 125: 8-9

16 Zanetta G, Rota S, Chiari S et al. Behaviour of borderline tumours with particular interest to persistence, recurrence, and progression to invasive carcinoma: a prospective study. J Clin Oncol 2001; 19: 2656-2664

17 Trillsch F, Mahner S, Vettorazzi E. Surgical staging and prognosis in serous borderline ovarian tumors (BOT): a subanalysis of the AGO ROBOT study. Br J Cancer 2015; 112: 660-666

18 Shim SH, Kim SN, Jung PS et al. Impact of surgical staging on prognosis in patients with borderline ovarian tumours: a meta-analysis. Eur J Cancer 2016; 54: 84-95

19 du Bois A, Ewald-Riegler N, de Gregorio $N$ et al. Borderline tumours of the ovary: a cohort study of the Arbeitsgemeinschaft Gynäkologische Onkologie (AGO) Study Group. Eur J Cancer 2013; 49: 1905-1914

20 Denschlag $D$, von Wolff $M$, Amant $F$ et al. Clinical recommendation on fertility preservation in borderline ovarian neoplasm: ovarian stimulation and oocyte retrieval after conservative surgery. Gynecol Obstet Invest 2010; 70: 160-165

21 Beiner ME, Gotlieb WH, Davidson B et al. Infertility treatment after conservative management of borderline ovarian tumours. Cancer 2001; 92: $320-325$

22 Fortin A, Morice P, Thoury A et al. Impact of infertility drugs after treatment of borderline ovarian tumours: results of a retrospective multicenter study. Fertil Steril 2007; 87: 591-596

23 Basille C, Olivennes $F$, Le C et al. Impact of gonadotropins and steroid hormones on tumour cells derived from borderline ovarian tumours. Hum Reprod 2006; 21: 3241-3245

24 von Wolff $M$, Thaler CJ, Frambach $T$ et al. Ovarian stimulation to cryopreserve fertilized oocytes in cancer patients can be started in the luteal phase. Fertil Steril 2009; 92: 1360-1365

25 Simi G, Obino ME, Casarosa E et al. Different stimulation protocols for oocyte cryopreservation in oncological patients: a retrospective analysis of single university centre. Gynecol Endocrinol 2015; 31: 966-970

26 Nekkebroeck J, Stoop D, Devroey P. A preliminary profile of women opting for oocyte cryopreservation for non-medical reasons. Hum Reprod 2010; 25: i15-i16

27 Rosendahl M, Greve T, Andersen CY. The safety of transplanting cryopreserved ovarian tissue in cancer patients: a review of the literature. J Assist Reprod Genet 2013; 30: 11-24

28 Fain-Kahn V, Poirot C, Uzan C et al. Feasibility of ovarian cryopreservation in borderline ovarian tumours. Hum Reprod 2009; 24: 850-855

29 Donnez J, Dolmans MM, Pellicer J et al. Fertility preservation for age-related fertility decline. Lancet 2015; 385: 506-507

30 Lotz L, Montag M, van der Ven $\mathrm{H}$ et al. Xenotransplantation of cryopreserved ovarian tissue from patients with ovarian tumors into SCID mice-no evidence of malignant cell contamination. Fertil Steril 2011; 95: 2612-2614

31 Cho HY, Kyung MS. CA 19-9 as a predictor of malignancy in primary ovarian mucinous tumors: a matched-case control study. Med Sci Monit 2014; 20: 1334-1339

32 Barnhill DR, O'Connor DM. Management of ovarian neoplasms of low malignant potential. Oncology (Williston Park) 1991; 5: 21-26; discussion $26,28,31-32$ 